\title{
Threshold effects of inflation on economic growth in developing countries
}

\author{
Alexander Bick *† \\ Department of Economics, Goethe University Frankfurt
}

\begin{abstract}
This paper introduces a generalized panel threshold model by allowing for regime intercepts. The empirical application to the relation between inflation and growth confirms that the omitted variable bias of standard panel threshold models can be statistically and economically significant.
\end{abstract}

Keywords: Panel threshold model, Inflation thresholds, Economic growth

JEL classification: C51, E31, O40

*Alexander Bick; House of Finance H28, Grueneburgplatz 1, 60323 Frankfurt, Germany; Tel.: +49 69 798 33829; Fax: +49 69798 33925; E-mail address: bick@wiwi.uni-frankfurt.de

${ }^{\dagger}$ I thank Bruce Hansen and Dieter Nautz for helpful comments and suggestions. Stephanie Kremer provided excellent research assistance. Financial support by the Monetary Stability Foundation is gratefully acknowledged. 


\section{Introduction}

A central objective of macroeconomic policies is to foster economic growth and to keep inflation on a low level. In recent years there has been substantial empirical work on the relationship between inflation and growth, yet the results have been mixed. Fisher (1993) was the first to identify a non-linear relationship where low inflation rates have a positive impact on growth which turns negative as inflation increases. Bruno and Easterly (1998) confirm the finding of a negative effect for high inflation rates but doubt the growthenhancing effect of low inflation. In line with this result, Khan and Senhadji (2001) estimate a threshold of $11 \%$ for developing countries where inflation rates above this threshold are associated with a significant negative effect on growth, while inflation rates below $11 \%$ do not have any significant impact.

This paper sheds new light on the inflation-growth nexus introducing a natural extension of Hansen's (1999) panel threshold model by accounting for regime intercepts. The empirical results confirm the importance of including a regime intercept from a statistical and economical perspective. Once the regime intercept is included, the threshold, up to which inflation is growth enhancing, decreases substantially and, more importantly, the negative impact of inflation above the threshold becomes significant.

The paper is structured as follows. The next section briefly reviews panel threshold estimation and discusses the role of regime intercepts. Section 3 introduces the data and presents the estimation results for the inflation-growth nexus. Finally, section 4 concludes.

\section{Panel threshold models}

Hansen (1999) proposes an estimation and inference strategy for balanced panels with individual specific effects and observations $\left\{y_{i t}, q_{i t}, x_{i t} ; 1 \leq i \leq N, 1 \leq t \leq T\right\}$ where the subscripts $i$ and $t$ index the individual and time. The equation of interest with one potential threshold $\gamma$ is given by

$$
y_{i t}=\mu_{i}+\beta_{1}^{\prime} x_{i t} I\left(q_{i t} \leq \gamma\right)+\beta_{2}^{\prime} x_{i t} I\left(q_{i t}>\gamma\right)+\varepsilon_{i t} \text { with } \varepsilon_{i t} \stackrel{i i d}{\sim}\left(0, \sigma^{2}\right)
$$

where $I(\cdot)$ is the indicator function and the threshold variable $q_{i t}$ divides the observations into two 'regimes' distinguished by differing regression slopes $\beta_{1}$ and $\beta_{2}$. The dependent 
variable $y_{i t}$ and $q_{i t}$ are both scalar. The latter may, but has not to be an element of $x_{i t}$, the $k$-dimensional vector of exogenous regressors 1 Moreover, some elements of $x_{i t}$ can be constrained to have the same impact in both regimes, i.e. $\beta_{1, j}$ may be equal to $\beta_{2, j}$ for some $j \in\{1, \ldots, k\}$. The individual specific effects are eliminated using the standard fixed-effects transformation implying for the identification of $\beta_{1}$ and $\beta_{2}$ that the elements of $x_{i t}$ are neither time-invariant nor adding up to a vector of ones. This latter case applies to regime intercepts which are usually included in each regime in threshold models in pure cross-sectional or time-series contexts. Even in the presence of fixed-effects it is possible to control for differences in the regime intercepts by including them in all but one regime as in the following extension of equation (1):

$$
y_{i t}=\mu_{i}+\beta_{1}^{\prime} x_{i t} I\left(q_{i t} \leq \gamma\right)+\delta_{1} I\left(q_{i t} \leq \gamma\right)+\beta_{2}^{\prime} x_{i t} I\left(q_{i t}>\gamma\right)+\varepsilon_{i t} .
$$

This formulation assumes that the difference in the regime intercepts, represented by $\delta_{1}$, is not individual specific but the same for all cross-sections. Since (2) has neither been considered by Hansen (1999) nor any of the numerous studies, e.g. Adam and Bevan (2005), Lensink and Hermes (2004) or Nautz and Scharff (2006), applying his methodology, it seems worthwhile to briefly discuss the role of regime intercepts for the estimation results in the Hansen (1999) framework 2

For any given threshold $\gamma$, the slope coefficients $\beta_{1}$ and $\beta_{2}$ can be estimated by ordinary least squares (OLS) using the data after the fixed-effects transformation. In case a regime intercept is included, as in specification (2), the slope estimates for each regime are identical to those from a regression using only observations from the respective regime which reflects the orthogonality of the regressors $x_{i} I\left(x_{i} \leq x_{m}\right)$ and $x_{i} I\left(x_{i}>x_{m}\right) 3$ Omission of any variable correlated with at least one regressor and the dependent variable causes biased estimates, but regime intercepts are a particularly interesting case. First, the bias can be clearly interpreted. Estimating equation (1) in the presence of a regime intercept in the data generating process results in a bias proportional to $\hat{\delta}_{1}$ because the orthogonality

\footnotetext{
${ }^{1}$ Caner and Hansen (2004) develop instrumental variable estimation for threshold models with endogenous regressors, but only for cross-sectional data.

${ }^{2}$ For a detailed review of the general estimation and inference strategy and the treatment of multiple thresholds the reader is referred to Hansen (1999).

${ }^{3}$ The exact algebraic expressions for the coefficient estimates of both specifications are given in the appendix which also lists the modifications needed in the setup considered by Hansen (1999) to allow for regime intercepts as in equation (2).
} 
of the regressors is not preserved anymore. Second, availability of regime intercepts as regressors is not an issue since they are as easily constructed as the regime-dependent exogenous regressors for a given threshold.

Biased estimates of the regression slopes have further consequences in the panel threshold model because the threshold estimates are also obtained by least squares:

$$
\hat{\gamma}=\underset{\gamma}{\operatorname{argmin}} S_{1}(\gamma)
$$

where $S_{1}(\gamma)$ is the sum of squared residuals from estimating (11) or (2) for a given threshold $\gamma$. Only by coincidence, these estimates will be the same for specifications (11) and (2) if a regime intercept is present in the data generating process. Moreover, unbiased estimates of $\beta_{1}$ and $\beta_{2}$ are crucial for the test of the significance of a threshold which can be represented by the following linear constraint

$$
H_{0}: \beta_{1}=\beta_{2}
$$

\section{The inflation-growth nexus}

The relationship between inflation and growth is investigated for a balanced panel of 40 developing countries through the period from 1960 to 2004 . As it is standard in the empirical growth literature, the results on the determinants of long-term economic growth will be based on five-year averages. The equation of interest is given by

$$
\Delta \ln g d p_{i t}=\mu_{i}+\beta_{1} \tilde{\pi}_{i t} I\left(\tilde{\pi}_{i t} \leq \gamma\right)+\delta_{1} I\left(\tilde{\pi}_{i t} \leq \gamma\right)+\beta_{2} \tilde{\pi}_{i t} I\left(\tilde{\pi}_{i t}>\gamma\right)+\phi^{\prime} w_{i t}+\varepsilon_{i t}
$$

representing a single threshold model that already includes a regime intercept. The dependent variable is the growth rate of GDP per capita. The inflation variable $\tilde{\pi}$ serves as the regime-dependent regressor and threshold variable and is a semi-log transformation of inflation with $\tilde{\pi}_{i t}=\pi_{i t}-1$, if $\pi_{i t}<1$ and $\tilde{\pi}_{i t}=\ln \pi_{i t}$, if $\pi_{i t} \geq 1$. Inflation rates smaller one are re-scaled for the sake of continuity. Using inflation levels in growth regressions implies that the marginal effect of inflation on economic growth is independent of the average level of inflation whereas the log model has the more plausible implication that multiplicative inflation shocks will have identical effects. The control variables are selected in accordance with the empirical growth literature, see e.g. Islam (1995) or Khan and Senhadji (2001), and passed the robustness tests in Levine and Renelt (1992), and Sala-i-Martin (1997). 
$w_{i t}$ contains investment as a share of GDP $(i g d p)$, population growth $(d p o p)$, the log of initial income per capita of the previous period (initial) as well as the growth rate and standard deviation of terms of trade (dtot, sdtot).

Table 1 presents the results for both specifications, i.e. without (column 1) and with (column 2) regime intercepts. The upper panel shows that in both cases the null hypothesis of no threshold can be rejected at the $5 \%$ significance level, while the presence of one threshold cannot be rejected. Inclusion of a regime intercept decreases the threshold estimate (middle panel) from 19\% to $12 \%$ and the lower bound of the $95 \%$ confidence interval from $11.8 \%$ to $5.3 \%$. The most striking point is that in absence of a regime intercept, inflation rates below the threshold of $19 \%$ have a significant positive effect (0.407) on growth only on the $10 \%$ significance level, while the negative impact (-0.232) for inflation rates above $19 \%$ is not statistically significant at all, compare the lower panel. In contrast, allowing for differences in the regimes' intercepts doubles the magnitude $(0.785$, -0.531) and establishes significance at least on the $5 \%$ level of the marginal impacts of inflation on growth in both regimes. The regime intercept $\hat{\delta}_{1}$ itself is also significant on the $5 \%$ level. Most of the regime-dependent coefficients are consistent with the implications of standard growth theory and are very similar for both specifications. The results from the specification with a regime intercept are in line with those by Khan and Senhadji (2001), despite that, similarly to Fisher (1993), low inflation rates (less than 12\%) are associated with a significant positive effect on growth 4

From a policy perspective, choosing the correct specification, i.e. controlling for differences in the regime intercepts, has important implications. First, the point estimate and lower bound of the confidence interval from which onwards inflation is harmful for growth are both substantially lower. Second, the detrimental impact for inflation rates above the threshold turns significantly and doubles in magnitude. Third, keeping inflation below the threshold has a stronger beneficial effect.

\footnotetext{
${ }^{4}$ The results of Khan and Senhadji (2001) are not exactly comparable to those presented here for two reasons. First, they use an unbalanced panel of more than 100 developing countries from 1960 to 1998. Second, they introduce continuity at the threshold which, though not explicitly stated, is nothing else but a nonlinear restriction on regime intercepts:$$
\Delta \ln g d p_{i t}=\mu_{i}+\beta_{1}\left(\tilde{\pi}_{i t}-\gamma\right) I\left(\tilde{\pi}_{i t} \leq \gamma\right)+\beta_{2}\left(\tilde{\pi}_{i t}-\gamma\right) I\left(\tilde{\pi}_{i t}>\gamma\right)+\phi^{\prime} w_{i t}+\varepsilon_{i t} .
$$

Note that the setup in Hansen (1999), with and without regime intercepts, implies a discontinuity at the threshold and refers to balanced panels.
} 


\section{Conclusion}

This paper revisits the relationship between inflation and economic growth for developing countries using a generalization of Hansen's (1999) panel threshold model. Regime intercepts are introduced and the potential bias of omitting these readily available regressors for both, regression slope and threshold estimates, is discussed. The regime intercept is significant in the inflation-growth nexus and affects the results in important ways.

\section{References}

Adam, C. S. and Bevan, D. L. (2005). Fiscal deficits and growth in developing countries, Journal of Public Economics 89: 571-597.

Bruno, M. and Easterly, W. (1998). Inflation Crisis and Long-Run Growth, Journal of Monetary Economics 41: 3-26.

Caner, M. and Hansen, B. E. (2004). Instrumental variable estimation of a threshold model, Econometric Theory 20(5): 813-843.

Fisher, S. (1993). The Role of Macroeconomic Factors in Growth, Journal of Monetary Economics 32: 485-512.

Hansen, B. E. (1999). Threshold Effects in Non-Dynamic Panels: Estimation, Testing, and Inference, Journal of Econometrics 93: 345-368.

Islam, N. (1995). Growth Empirics: A Panel Data Approach, The Quarterly Journal of Economics 110(4): 1127-1170.

Khan, M. S. and Senhadji, A. S. (2001). Threshold effects in the Relationship between Inflation and Growth, IMF Staff Paper 48(1): 1-21.

Lensink, R. and Hermes, N. (2004). The short-term effects of foreign bank entry on domestic bank behaviour: Does economic development matter?, Journal of Banking \& Finance 28: 553-568.

Levine, R. and Renelt, D. (1992). A Sensitivity Analysis of Cross-Country Growth Regressions, American Economic Review 82(4): 942-963.

Nautz, D. and Scharff, J. (2006). Inflation and Relative Price Variability in the Euro Area: Evidence from a Panel Threshold Model, Deutsche Bundesbank Discussion Paper 14/06.

Sala-i-Martin, X. (1997). I just ran two million regressions, American Economic Review 87: $178-183$. 


\section{A Appendix}

\section{A.1 Coefficient estimates}

Without loss of generality assume that $T=1 ; \mu_{i}=\mu \forall i=1, \ldots, N ; x$ is scalar and $x_{1}<x_{2}<\ldots<x_{m}<x_{m+1}<\ldots<x_{N}$ and the threshold is known at $\gamma=x_{m}$ s.t. (1) and (2) boil down to

$$
y_{i}=\tilde{\mu}+\tilde{\beta}_{1} x_{i} I\left(x_{i} \leq x_{m}\right)+\tilde{\beta}_{2} x_{i} I\left(x_{i}>x_{m}\right)+\varepsilon_{i}
$$

and

$$
y_{i}=\mu+\beta_{1} x_{i} I\left(x_{i} \leq x_{m}\right)+\delta_{1} I\left(x_{i} \leq x_{m}\right)+\beta_{2} x_{i} I\left(x_{i}>\gamma\right)+\varepsilon_{i} .
$$

The coefficient estimates for specification (A.2) are given by

$$
\left(\begin{array}{c}
\widehat{\mu} \\
\widehat{\beta}_{1} \\
\widehat{\delta}_{1} \\
\widehat{\beta}_{2}
\end{array}\right)=\left(\begin{array}{c}
\frac{1}{N-m} \sum_{i=m+1}^{N} y_{i}-\widehat{\beta}_{2} \frac{1}{N-m} \sum_{i=m+1}^{N} x_{i} \\
\frac{1}{m} \sum_{i=1}^{m} x_{i} y_{i}-\frac{1}{m} \sum_{i=1}^{m} x_{i} \frac{1}{m} \sum_{i=1}^{m} y_{i} \\
\frac{1}{m} \sum_{i=1}^{m} x_{i}^{2}-\left[\frac{1}{m} \sum_{i=1}^{m} x_{i}\right]^{2} \\
\frac{1}{m} \sum_{i=1}^{m} y_{i}-\widehat{\beta}_{1} \frac{1}{m} \sum_{i=1}^{m} x_{i}-\widehat{\mu} \\
\frac{1}{N-m} \sum_{i=m+1}^{N} x_{i} y_{i}-\frac{1}{N-m} \sum_{i=m+1}^{N} x_{i} \frac{1}{N-m} \sum_{i=m+1}^{N} y_{i} \\
\frac{1}{N-m} \sum_{i=m+1}^{N} x_{i}^{2}-\left[\frac{1}{N-m} \sum_{i=m+1}^{N} x_{i}\right]^{2}
\end{array}\right)
$$

and can be expressed for specification (A.1) in the following way

$$
\left(\begin{array}{c}
\widehat{\widetilde{\mu}} \\
\widehat{\widetilde{\beta_{1}}} \\
\widehat{\widetilde{\beta_{2}}}
\end{array}\right)=\left(\begin{array}{c}
\widehat{\mu} \\
\widehat{\beta_{1}} \\
\widehat{\beta}_{2}
\end{array}\right)+\left(\begin{array}{c}
\frac{\sum_{i=m+1}^{N} x_{i}^{2}\left[\left(\sum_{i=1}^{m} x_{i}\right)^{2}-m \sum_{i=1}^{m} x_{i}^{2}\right]}{\left(\sum_{i=m+1}^{N} x_{i}\right)^{2} \sum_{i=1}^{m} x_{i}^{2}+\sum_{i=m+1}^{N} x_{i}^{2}\left[\left(\sum_{i=1}^{m} x_{i}\right)^{2}-N \sum_{i=1}^{m} x_{i}^{2}\right]} \\
\frac{\sum_{i=1}^{m} x_{i}\left[\left(\sum_{i=m+1}^{N} x_{i}\right)^{2}-(N-m) \sum_{i=m+1}^{N} x_{i}^{2}\right]}{\left(\sum_{i=m+1}^{N} x_{i}\right)^{2} \sum_{i=1}^{m} x_{i}^{2}+\sum_{i=m+1}^{N} x_{i}^{2}\left[\left(\sum_{i=1}^{m} x_{i}\right)^{2}-N \sum_{i=1}^{m} x_{i}^{2}\right]} \\
\frac{-\sum_{i=m+1}^{N} x_{i}\left[\left(\sum_{i=1}^{m} x_{i}\right)^{2}-m \sum_{i=1}^{m} x_{i}^{2}\right]}{\left(\sum_{i=m+1}^{N} x_{i}\right)^{2} \sum_{i=1}^{m} x_{i}^{2}+\sum_{i=m+1}^{N} x_{i}^{2}\left[\left(\sum_{i=1}^{m} x_{i}\right)^{2}-N \sum_{i=1}^{m} x_{i}^{2}\right]}
\end{array}\right) \widehat{\delta_{1}}
$$

where $\widehat{\beta}_{1}, \widehat{\beta}_{2}$ and $\widehat{\delta}_{1}$ are taken from (A.3). Note that in the presence of a fixed effect, $\widehat{\mu}$ and $\widehat{\widetilde{\mu}}$ would correspond to the estimate of the average fixed effect.

\section{A.2 Regime intercepts in the panel threshold model}

The setup in Hansen (1999) has to be extended to allow for regime intercepts as in equation

(2). First, the null hypothesis to test for the significance of the threshold has to be extended 
by $\delta_{1}=0$. Second, the derivation of the asymptotic distribution of the threshold estimate now relies on the additional technical assumption that $\delta_{1} \rightarrow 0$ as $N \rightarrow \infty$. It means that the difference in the intercepts between the two regimes is 'small' relative to sample size which is completely analogous to the assumption regarding the slope coefficients, namely that $\left(\beta_{2}-\beta_{1}\right) \rightarrow 0$ as $N \rightarrow \infty$. Third, the proof in the appendix now relies on the following two expressions taking the regime intercept as an additional regressor into account: $\theta^{\prime}=\left(\left(\beta_{2}-\beta_{1}\right)^{\prime}-\delta_{1}\right)$ and $z_{i t}^{\prime}=\left(\begin{array}{ll}x_{i t}^{\prime} & 1\end{array}\right) C$. 
Table 1: Inflation-growth nexus in developing countries

No regime intercepts

Regime intercepts

Test for the number of thresholds: p-value

\begin{tabular}{lll}
\hline$H_{0}:$ No threshold $(K=0)$ & 0.013 & 0.025 \\
$H_{0}:$ At most one threshold $(K=1)$ & 0.252 & 0.642
\end{tabular}

Threshold estimates and confidence intervals

\begin{tabular}{lcc}
\hline$\hat{\gamma}$ & $19.16 \%$ & $12.03 \%$ \\
$95 \%$ confidence interval & {$[11.82 \%, 20.48 \%]$} & {$[5.29 \%, 20.48 \%]$} \\
\hline \hline
\end{tabular}

Coefficient estimates: $\quad \Delta \ln g d p_{i t}=\mu_{i}+\beta_{1} \tilde{\pi}_{i t} I\left(\tilde{\pi}_{i t} \leq \gamma\right)+\delta_{1} I\left(\tilde{\pi}_{i t} \leq \gamma\right)+\beta_{2} \tilde{\pi}_{i t} I\left(\tilde{\pi}_{i t}>\gamma\right)+\phi^{\prime} w_{i t}+\varepsilon_{i t}$

Regime-dependent regressors

$\hat{\beta}_{1} \quad 0.407^{*}$

$0.785^{* * *}$

$\hat{\delta}_{1}$

$-1.985^{* *}$

$\hat{\beta}_{2}-0.232$

$(1.000)$

$-0.531^{* *}$

Regime-independent regressors

$\begin{array}{llc}\text { initial } & -3.353^{* * *} & -3.341^{* * *} \\ & (0.563) & (0.567) \\ \text { igdp } & 0.031 & 0.021 \\ & (0.041) & (0.042) \\ \text { dpop } & -0.814^{* * *} & -0.646^{* *} \\ & (0.306) & (0.307) \\ \text { dtot } & 0.014 & 0.002 \\ & (0.028) & (0.028) \\ \text { sdtot } & -0.054^{* *} & -0.052^{* *} \\ & (0.020) & (0.020)\end{array}$

Notes: Standard errors are given in parentheses, */**/*** indicate the $10 \% / 5 \% / 1 \%$ significance level. Similarly to Hansen (1999), each regime has to contain at least $5 \%$ of all observations. 1000 bootstrap replications were used to obtain the p-values to test for the number of thresholds. By construction, the confidence intervals for the threshold estimates can be highly asymmetric. The likelihood ratio statistics and critical values for determining the number of thresholds are available from the author upon request. The dataset and an extension of Bruce Hansen's program that accounts for regime intercepts can be downloaded from the author's website: http://www.wiwi.uni-frankfurt.de/profs/fuchs/bick.html. 